\section{PRINCIPLES OF PERCUSSION AND AUSCULTATION. ${ }^{1}$}

BY FRIEDRICH MÜLLER, M.D., LL.D., PROFESSOR OF CLINICAL MEDICINE AT THE UNIVERSITY OF MUNICH.

THOSE who have taught physical diagnosis have realised, I am sure, the difficulty of giving precise definitions of the conceptions of the percussion sound and the auscultatory symptoms. The usual clinical terms " clear," "flat," "dead," "full" are metaphorical and not coincident with the conceptions of scientific physics. They therefore give rise to much confusion. We shall overcome these difficulties only if we go down to the very principles of physics and use the rules and terms of science instead of the ambiguous expressions of clinical practice. The works of Helmholtz, of Tyndall, and of Geigel ${ }^{2}$ will give the best advice to all physicians who wish to deal more exactly with these problems of acoustics.

The varieties of sound are comprised in the following terms: 1. Loud and soft express the intensity or amplitude of the waves. 2. High and low or the pitch gives the number of waves in the second, and therefore the length of the single vibration. 3. Long and short mean the duration of the total sound. I propose not to use the term " timbre" or "colour" of sound, because this only depends on the combination of the fundamental tone with different overtones.

Difficulties will arise as soon as we proceed to analyse the sounds of percussion and auscultation, because the physical methods of analysing sounds have not been sufficiently worked out for our purpose. We were, therefore, obliged to find our own way. For the estimation of the intensity we have used a very thin rubber membrane armed with a minute mirror, which reflected a lioht ray on to a photographic film, or the microphone in combination with Einthoven's galvanometer. ${ }^{3}$ The same instruments give evidence of the total duration of the percussion sound. A simpler method of estimation is based upon the physical law, that the intensity of sound diminishes with the square of the distance. This law is employed in otology for measuring the acuity of hearing. We can apply the converse principle and estimate the intensity of sound by the distance over which it is still perceptible. A flat percussion sound will be distinguished only in the immediate neighbourhood of the patient; a loud sound in the most remote seats of a large amphitheatre.

We conclude from our estimations that the percussion sound of the normal lung and other air-containing organs shows, not only a far greater intensity, but also a longer duration than the flat sound of a solid organ. The tracings with the sensitive membrane or the Einthoven galvanometer show for the full sound of the lung an amplitude three or four times as great and a duration twice $(0.42$ to 0.28 second) as long as the flat sound. It is obvious that such a comparison is only permissible if the same experimental. conditions are kept-i.e., if the percussion stroke is of the same kind and intensity. But the conception of "dulness" ("matité" of Laënnec) is not fully identical with small amplitude and short duration. We realise that the problem is more complicated as soon as we proceed to consider the pitch.

In the first place we found great difficulty in analysing the pitch. The microphone proved to be unfit, because it was accompanied by too many errors and because it did not respond with the lower tones. The direct registration by sensitive membranes (rubber membranes or soap bubbles) and transmissions of its vibrations by a ray of light to a photographic film give beantiful drawings, ${ }^{4}$ but it is often

1 This paper reproduces in substance a clinical lecture delivered at Guy's Hospital on June 29th, 1911, but only now received for publication in England.

2 John Tyndall: Sound, German edition by Helmholtz. Richard Geigel : Leitfaden der diagnostischen Akustik, Stuttgart, Verlag von F. Enke, 1908. H. von Helmholtz : Die Lehre von den Tonempfindungen, Braunsch weig, 1896.

$3 \mathrm{Th}$. Selling : Untersuchungen über den Perkussionsschall, Deutsches Archiv für Klinische Medizin, Band xc., p. 163, 1907. F. Mïlller : Der Ausbau der Klinischen Untersuchungsmethoden, Zeitschrift für Airtliche Fortbildung, 3. Jahrgang, 1906. Idem: Zur Physikalischen Diagnostik, Verhandlungen des Deutschen Kongresses für Innere Medizin, Wiesbaden, 1911.

4 May und Lindemann: Graphische Studien iiber den Percussionsschall, Deutsches Archiv fur Klinische Medizin, Band xciii., p. 100. difficult to determine which part of the waves is dependent. upon the fundamental tone of the membrane and which is the true expression of the given percussion sound. This method is not yet worked out perfectly, and it gives. good results only if the sound is loud enough. We got the best results by the use of resonators. The spherical resonators of Helmholtz did not respond so clearly as those of conical form, to which Professor Röntgen has drawn my attention. The conical tubes were manufactured from tin. plate. The length of the cone is four or five times as great as the diameter of the base. The apex of the cone shows a lumen of 2 millimetres and is applied directly to one ear, or by the tube of a binauricular stethoscope to both ears. The keynote of each cone is identical with that of a cylinder open at both ends--i.e., the conical resonator responds to a tone of double its wave length. We use a long series of such cones, the length of which varies from 10 centimetres to 3 metres. The application of such a long series of single resonators offers some inconvenience, and we therefore proceeded to use resonators of cylindrical shape. Two or more cylindrical tubes were fitted into one another like a telescope; one end of the inner tube is closed and perforated by a small opening of 2 millimetres upon which the tube of a binauricular stethoscope can be fitted. The length, and therefore the keynote, of these cylindrical resonators can be changed easily, and their use has great advantages. A cylinder closed at one end responds to a tone of four time its wavelength. If the tube is applied with its open end to the chest wall for the purpose of auscultation, it follows the laws of cylinders closed at both ends and responds to a tone of twice its wave length-i.e., to the higher octave. The diameter of the tubes is of little importance. We use cylinders with a diameter of 3 or 5 centimetres. An experienced ear is easily able to distinguish with what length of the tube resonance takes place. The length of the tube expressed in centimetres permits one to read directly the pitch of the tone.

It is necessary to remember that the low fundamental tone of a resonator never can be evoked by a higher tone, but that a high tone is able to evoke the high partial tone of a big resonator. With the help of the resonator tubes we have been able to ascertain that the percussion sound of the normal lung is characterised by a very low note of the contra octave (especially A) or the upper half of the subcontra octave. Very often we found the best resonance with a length of the tube of 1.6 to 1.8 metres-that is, $A_{1}$ to $G$

The foam-like substance of the normal lung answers with very low and slow vibrations to the stroke of the percussor, and we conclude that the fundamental tone of the normal lung is very low. But this very low sound is only heard if the lung is fully expanded with air and if the part set into. vibration is great enough. The thinner edges of the base of the lung near the diaphragm or covering the heart give a higher pitch. The apex of the lung gives, too, a distinctly higher pitch than the lower parts, especially on the back below the scapula. The lung of a child gives a higher note than that of an adult; the emphysematous lung and the pneumothorax the lowest.

You may convince yourself of the resonant quality of the lungs by a very simple experiment. Put your hands on both sides of your own chest and sing the scale. You will feel no vibrations as long as you sing the upper parts of the scale. From a certain point of the scale vibrations of the chest wall become perceptible, and are strongest with the lower notes of the bass voice. My assistant, Dr. Edens, was able to record these vibrations of the chest wall with Otto Franks's sphygmograph, and has shown that the figure of thoracic vibrations exactly corresponds with the oscillations of the sungen tone. We may conclude that the lung is set into vibration like a Helmholtz resonator as soon as its fundamental tone is emitted. We understand why pectoral fremitus is commonly not distinctly perceptible on the female chest wall. The female lung has nearly the same low fundamental tone as the male, but the female voice is not suited to emit the low keynotes which only find resonance in the lung tissue.

The tympanitic percussion sound of the air-containing intestines is of distinctly higher pitch than that of the lungs; it responds to an open resonator of from 30 to 50 centimetres length-i.e., from $D$ to $\mathbf{E}$ of the scale. The tympanitic sound is characterised by a simpler constitution. 
May and Lindemann have been able to trace its vibration by means of a soap membrane, and found that it nearly corresponded to a single tone, while the percussion sound of the normal lung gives a very complicated diagram made up of various higher, and especially lower, tones.

The dulness of the infiltrated lung (pneumonia) or pleuritic effusions shows not only a very small amplitude of the vibrations but also a deficiency of the lower tones. It is not only soft but higher too. And because the iower tones always have a longer duration than the higher ones, the percussion sound in case of dulness is quicker extinguished (damped) and has a shorter total duration than the sound of the normal lung.

Though the total duration of the percussion sound is always rather short, amounting only to half a second or less, the ears of many physicians are especially adapted to perceive the duration, and they therefore use the expression "short" instead of "dull," not without good reason. In beginning tuberculosis of the lung the percussion sound of the affected apex at first shows only the deficiency of the lower tones, and therefore appears in toto higher and shorter. A difference of amplitude (loudness) is scarcely or not at all perceptible in early cases ; a distinctly smaller amplitudei.e., real dulness or flatness-is a sign of more intense infiltration.

The physical analysis of the auscultatory phenomena presented greater difficulties than that of the percussion sound. The intensity of the respiratory sounds is so very small that our apparatus up to the present time proved unable to record its vibrations. Only the most sensitive of all acoustic apparatus, the human ear, is fit to perceive it fully. We first tried to use microphones and telephones of different types. But they idid not respond to the sound of normal vesicular breathing, but only to that of bronchial and tracheal breathing. An exact exploration by the scale of tuning forks gave evidence that the telephone and microphone do not respond to low tones as well as to high ones, -or to the lower tones not at all if they are of low amplitude. This experiment led to the conclusion that bronchial breathing was made up of high tones only, and that in vesicular breathing high tones are not present, but only low ones. An exploration by resonating tubes of different length proved that this assumption was right. Normal vesicular breathing gives only resonance with tubes of 120 to 300 centimetres length (c to $A_{1}$ ), bronchial breathing with tubes of 26 to 60 centimetres $\left(\mathrm{F}^{\prime \prime}\right.$ to $\left.\mathrm{D}^{\prime}\right)$. Consonating râles in infiltrated lung resonate on an average in the same part of the scale as bronchial breathing, partly with higher tones. The metallic sound in percussion as well as in auscultation (amphoric breathing, metallic resonant râles) are characterised by very high overtones perhaps of 1000 to 5000 waves in the second.

We must make the conception of bronchial breathing as clear as possible. Real bronchial breathing is heard over complete pneumonic infiltrations or over totally compressed atelectatic lungs-i.e., on the upper parts of pleuritic effusions. This characteristic breathing sound consists of only high tones, and resembles exactly the sharp ch sound of the German language. The so-called bronchial breathing over tuberculous cavities commonly shows a lower pitch. Tracheal breathing is not at all coincident with bronchial breathing, as is often said. The breath sound over the trachea shows especially the high pitch of a tube of the same diameter, but this sound is mixed with the low sound of vesicular breathing. The bronchi which ramify into the interior of the lungs conduct the vesicular breathing sound to the trachea. We can convince ourselves very easily of this fact if we practise the auscultation of the trachea with the help of a long rubber tube-viz., a gas tube averaging 2 metres in length. The long air tube between the trachea and the ear deadens or damps the high tones of the tracheal breathing nearly to complete extinction, and the low tones of the pre-existent vesicular breathing alone remain perceptible. This experiment proves that the interposition of an air space damps the higher waves more than the low ones, and a solid bar of wood or iron conducts the higher sound waves far better than an air tube of the same length.

The spongy substance of the normal lung seems to have a far more damping effect upon high sound, than air alone; it may be because the manifold reiteration of air bubbles and the walls of the lung vesicles give rise to reflection and extinction of the waves. A comparatively small layer of lung tissue deadens and extinguishes the high pitch of the underlying tracheal and bronchial tubes almost completely, and only in the immediate neighbourhood of the trachea and the principal bronchi are we able to hear a trace of its characteristic high breathing sound. In the lower parts of the lung no trace at all of the bronchial breathing sound is heard. The normal lung extinguishes, too, the sound of the heart over a short distance. Only if the lung is consolidated by inflammation, by neoformation of connective tissue, or by compression does its conducting power increase and equal the conducting power of solid masses, and under these conditions only are we able to perceive on the chest wall the high whistling sounds which originate in the bronchi. Characteristic bronchial breathing seems to originate only in the greater bronchi of the first, second, and third order. The smaller bronchi seem from our experiments to be too narrow to give origin to perceptible sounds.

It is not easy to give a convincing explanation of the origin of the normal vesicular breathing sound. It is likely that the stretching and successive extension of the lung tissue give origin to vibration of the lung substance as a whole and therefore to the low characteristic sound. A comparison between the size of the lun in the living body and the contracted organ after it has been removed at a necropsy shows clearly that the lung must be very greatly expanded in the normal thoracic cavity, and this expansion increases with every inspiration.

I have tried to give a picture of the results of our experiments in the following scale, and we may conclude from the scale that the normal lung responds on an average with the same low sound, if its fundamental tone is evoked by percussion or by the stretching of inspiration or by the voice. The fundamental tones of the cavities of the abdomen have a higher rate of vibration; the fundamental tone of the bronchi (bronchial breathing, consonant râles) takes its place between $\mathrm{C}^{\prime}$ and $\mathrm{C}^{\prime \prime}$; and the metallic sound is ehararterised by the highest overtones. The normal heart sounds and the murmurs of valvular diseases show the same pitch as vesicular breathing-i.e., between 60 and 160 waves in the second and therefore it is sometimes very difficult to distinguish systolic vesicular breathing from valvular murmurs.

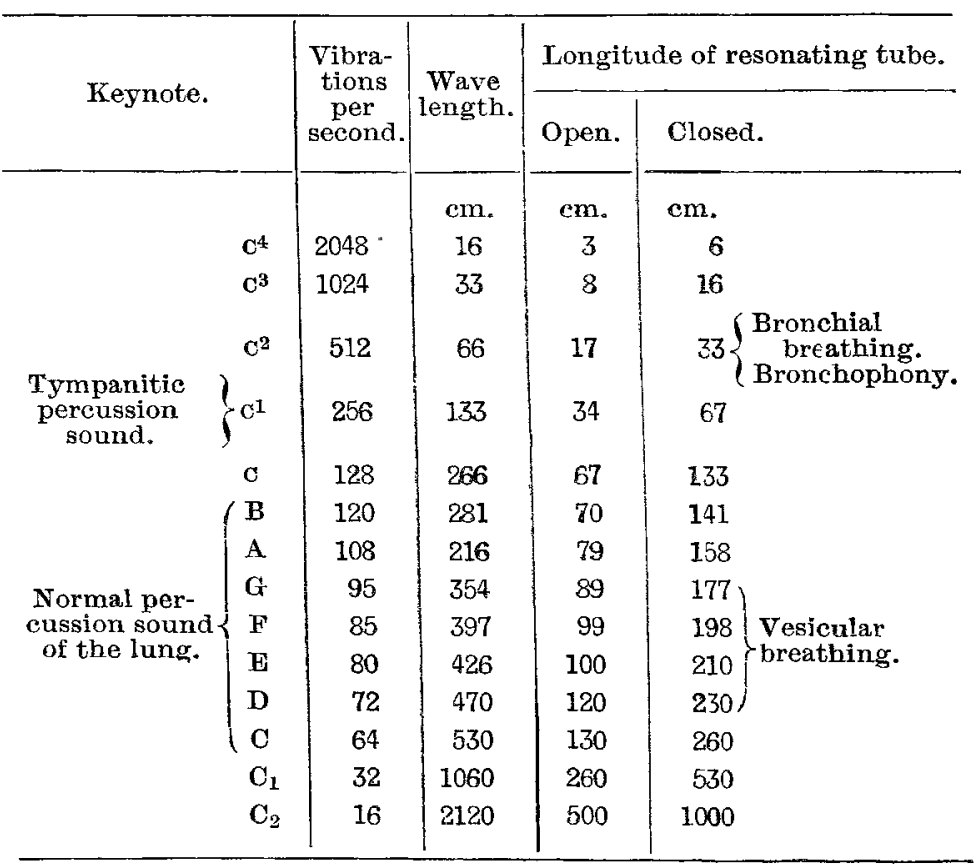

Some physicians have tried to exaggerate the technical refinement of percussion and auscultation to artistic virtuosity, and to draw the keenest diagnostic conclusions from its application. I have tried to show that these methods of examination are not so much the object of artistic skill, but of scientific understanding, and that we first need a solid scientific foundation and criticism. A comparison between the results of percussion and auscultation with the results of the Roentgen ray exploration or the necropsy finding often shows that we have to be very moderate in diagnostic conclusions from the physical examination, and that it does not grant so conclusive proof concerning the conditions of the inner organs as many of us have imagined. 